$63^{\text {ème }}$ Congrès de la SFCO, 03037 (2015)

DOI:10.1051/sfco/20156303037

(C) Owned by the authors, published by EDP Sciences, 2015

\title{
Etude du micro-environnement immunitaire des carcinomes épidermoïdes de la cavité orale (MICECO) : présentation du projet
}

\author{
Rochefort J***, Guillot-Delost M*, Macedo R*, Baillou C*, Poulain $\mathrm{C}^{* * * *}$, Tanaka $\mathrm{K}^{*}$, \\ Bertolus $\mathrm{C}^{* * * * *}$, Lemoine $\mathrm{F}^{* * * *}$, Lescaille $\mathrm{G}^{* * * *}$ \\ * Sorbonne Universités, UPMC Univ-Paris 06, CIMI-Paris - INSERM U 1135, CNRS ERL 8255, Paris \\ ** AP-HP, Groupe hospitalier Pitié-Salpêtrière, service Odontologie, unité de Chirurgie Buccale, Paris \\ *** AP-HP, Groupe hospitalier Pitié-Salpêtrière, service de Stomatologie et Chirurgie Maxillo-Faciale, Paris
}

Les cancers des VADS représentent le $5^{\text {ème }}$ cancer le plus fréquent en France. 20 à $25 \%$ de ces cancers sont situés au niveau de la cavité orale, 90 \% d'entre eux sont des carcinomes épidermoïdes (Parkin et al. 2005). Des observations cliniques semblent indiquer des différences de réponses aux traitements conventionnels (chirurgie, radiothérapie et chimiothérapie) parmi les patients ayant ou non des facteurs de risque connus (intoxication alcoolo-tabagique). C'est pourquoi il est important d'étudier le micro environnement tumoral de différents groupes de patients atteints de cancer epidermoïde de la cavité orale (CECO) afin de mieux identifier les interactions entre les cellules tumorales et le système immunitaire jouant un rôle clef dans les défenses anti-tumorales (Gavin P. Dunn 2004). De telles études ont déjà été réalisées pour d'autres cancers (mélanome, cancers du côlon, cancers de l'oropharynx). Elles portent pour la plupart sur l'analyse d'échantillons sanguins ; peu ont étudié l'infiltrat immunitaire tissulaire. Une identifie comme facteur de bon pronostic l'augmentation des Lymphocytes T régulateurs tumoraux dans les cancers des VADS (Badoual et al. 2013).

Notre étude s'inscrit dans une démarche théranostique, c'est à dire l'identification de nouveaux biomarqueurs diagnostiques et pronostiques destinés à constituer à terme de nouvelles cibles thérapeutiques.

Les objectifs sont d'étudier, dans le sang et le microenvironnement tumoral de patients atteints de CECO, les modifications quantitatives et qualitatives des populations immunitaires selon le stade de la maladie et/ou les facteurs de risque. Les prélèvements sont réalisés avant tout traitement et au moment de la première chirurgie d'exérèse. En utilisant des techniques de cytométrie en flux completées par des analyses immuno-histochimiques et de biologie moléculaire, nous étudions les cellules de l'immunité innée (polynucléaires neutrophiles [PN], monocytes et cellules Natural Killer [NK]) et de l'immunité adaptative (lymphocytes T et B). Parmi les lymphocytes T (populations CD4 et CD8), sont analysés les sous-populations mémoires, naïves, les populations dites lymphocytes $\mathrm{T}$ régulatrices (Treg) ainsi que la polarisation des Lymphocytes $\mathrm{T}$ effecteurs et plus précisément les Th17. Les données cliniques des patients sont collectées au moment de leur inclusion. Les données sont comparées à un groupe temoins : prélèvements tissulaires sains provenant de déchets opératoires obtenus lors d'actes chirurgicaux d'avulsion des troisièmes molaires; prélèvements sanguins issus de sujets sains et/ou de patients alcoolo-tabagiques non cancéreux (Département d'addictologie, Service de Pneumologie, Pitié-Salpêtrière). Les données cliniques des patients sont collectées au moment de leur inclusion.

This is an Open Access article distributed under the terms of the Creative Commons Attribution License 4.0, which permits unrestricted use, distribution, and reproduction in any medium, provided the original work is properly cited. 
Ce projet bénéficie au sein de l'hôpital de la Pitié Salpêtrière des interactions étroites entre le service de Chirurgie Maxillo-Faciale (Dr. Chloé Bertolus) qui dispose d'un recrutement important (150 nouveaux cas par an), le service d'Odontologie (Dr. Geraldine Lescaille) et l'équipe de recherche du Pr. F. Lemoine située au sein d'une unité labellisée CNRS/INSERM. Les résultats préliminaires seront exposés lors de la présentation.

Nom et adresse du conférencier

\section{Juliette ROCHEFORT}

Service Odontologie, Hôpital Pitié Salpêtrière

47-83 Bd. de l'hôpital

75013 Paris (France)

julietterochefort@yahoo.fr 